\title{
Conduct Disorder and Alcohol Use Disorder Trajectories, Predictors, and Outcomes for Indigenous Youth
}

\author{
Brenna L. Greenfield, PhD, Kelley J. Sittner, PhD, Miriam K. Forbes, PhD, \\ Melissa L. Walls, PhD, Les B. Whitbeck, PhD
}

Objective: The aim of this study was to identify separate and joint trajectories of conduct disorder (CD) and alcohol use disorder (AUD) DSM-IV diagnostic symptoms among American Indian and First Nation (Indigenous) youth aged 10 to 18 years, and to characterize baseline profiles and later outcomes associated with joint trajectory group membership.

Method: Data were collected between 2002 and 2010 on three indigenous reservations in the northern Midwest and four Canadian reserves $(\mathrm{N}=673)$. $\mathrm{CD}$ and substance use disorder (SUD) were measured using the DSM-IV Diagnostic Interview Schedule for Children-Revised (DISC-R), administered at four time points.

Results: Using group-based trajectory modeling, three CD and four AUD trajectories were found. Both had a small group with high symptoms, but the largest groups for both had no symptoms (55\% and $73 \%$, respectively). CD symptom trajectories began at age 10 years and peaked at age 14; AUD trajectories began at age 12 years and were highest from age 16 on. Eight joint trajectories were identified. Of the sample, 53\% fell into the group with no CD or AUD symptoms. Compared to symptomatic groups, this group had greater caretaker warmth, positive school adjustment, less discrimination, and fewer deviant peers, and were less likely to have a caretaker with major depression at baseline. Symptomatic groups had higher odds of high school dropout, sex under the influence, and arrest at age 17 to 20 years.

Conclusion: Despite significant risk factors, a large proportion of Indigenous youth had no CD-SUD symptoms over time. CD-SUD symptoms have multiple development trajectories and are related to early developmental risk and later psychosocial outcomes.

Key words: alcohol use disorder, conduct disorder, American Indian, First Nations, comorbidity

J Am Acad Child Adolesc Psychiatry 2017;56(2):133-139. onduct disorder (CD), characterized by rule-breaking and aggressive behavior, is associated with a variety of negative individual and societal outcomes, including earlier initiation of alcohol use and higher rates of alcohol use disorder (AUD) ${ }^{1-3}$ Past-year prevalence of CD was $5.4 \%$ in the school-based National Comorbidity Survey Replication-Adolescent Supplement (NCS-A), ${ }^{4}$ a sample that was $56 \%$ non-Hispanic white, and included adolescents aged 13 to 17 years. Although CD can develop into adult antisocial personality disorder, many individuals' CD behaviors dissipate as they enter adulthood, and others have emphasized the importance of distinguishing between adolescent-limited versus life course-persistent antisocial behavior groups. ${ }^{5}$

In general population surveys, higher rates of $C D$ have been reported in racial/ethnic minority groups and those from lower socioeconomic (SES) backgrounds. ${ }^{2,6}$ Early environmental factors that predict $C D$ at ages 14 to 16 years include low SES, childhood adversity, parental substance use

This article is discussed in an editorial by Drs. Nancy Rumbaugh Whitesell and Carol E. Kaufman on page 103.

Supplemental material cited in this article is available online. disorder (SUD), negative home environment, and delinquent peers. ${ }^{7}$ American Indian (AI) and other Indigenous youth are disproportionately exposed to these poverty- and family adversity-related risks, ${ }^{8}$ in large part due to current and historical factors (e.g., forced relocation, boarding schools, social marginalization), and thus may be particularly at risk for $\mathrm{CD}$ and related negative outcomes. In the longitudinal study of AI and First Nation (hereafter "Indigenous") adolescents that forms the basis of this article, risk factors for CD at age 14 years included concurrent discrimination, delinquent peers, and prior CD or oppositional defiant disorder (ODD) diagnosis. ${ }^{9}$ Higher rates of incarceration, biases in diagnosis, and poorer access to mental health treatment compound these racial and ethnic disparities. ${ }^{10}$

Despite their greater exposure to CD risk factors, AI youth have not been found to have substantially elevated CD rates compared to other youth. For example, in the Great Smoky Mountain Study, white and Cherokee youth aged 9 to 13 years in Appalachia had similar 3-month prevalence rates of CD or ODD (6.5\% Cherokee; $5.3 \%$ white). ${ }^{8}$ Among Northern Plains $\mathrm{AI}$ adolescents, the 6-month prevalence of $\mathrm{CD}$ was $3.8 \%$ between ages 14 and 17 years and did not differ significantly from a general population sample. ${ }^{11}$ In the present sample, past-year prevalence of CD has been reported to peak at age 14 years at $15.7 \%$ and decrease to $1.3 \%$ by age 18 years. $^{12}$ 
Conduct disorder is most commonly paired with other externalizing disorders, particularly SUD. ${ }^{11,13}$ This comorbidity is considered to be a reflection of underlying aspects of CD and SUD, such as the prominence of disinhibition. ${ }^{14}$ Both disorders are classified under the umbrella of externalizing disorders, and there is evidence that they have a shared genetic liability. ${ }^{15}$ Furthermore, within the externalizing spectrum model, comorbid disorder presentation is associated with higher severity, poorer treatment response, and more negative outcomes. ${ }^{16,17}$

No study has examined how these disorders unfold together over the key developmental period of adolescence. This study fills that gap by considering joint trajectories of CD and SUD comorbidity (and, more specifically, AUD)—or lack thereof-among Indigenous individuals aged 10 to 18 years. Based on diagnostic symptom counts, we used groupbased trajectory modeling to determine trajectory groups of comorbid CD and AUD (i.e., taking into account both CD and AUD symptom development over time), to identify risk factors that prospectively predict trajectory group membership, and to characterize their associated age-18 outcomes. We focused on AUD symptoms because Indigenous adults have higher rates of AUD and alcohol-related consequences than other racial and ethnic groups, ${ }^{18}$ yet lower and/or similar rates of alcohol use. ${ }^{19}$

\section{METHOD}

\section{Participants and Procedure}

These data were collected as part of a longitudinal study on three reservations in the northern Midwest and four Canadian reserves between 2002 and 2010. The project was designed in partnership with the participating reservations and reserves. Before the application funding, the research team was invited to work on these reservations, and tribal resolutions were obtained. The university institutional review board also approved all procedures. At each participating location, an advisory board was appointed by the tribal council to provide guidance and oversight of the research process, including review of publications. Data from one reservation are not included because that location did not have an active advisory board, and we wanted to respect the community's right to review research prior to publication.

Each of the participating reservations and reserves provided a list of families of tribally enrolled individuals aged 10 to 12 years who lived on or within 50 miles of the reservation or reserve. We attempted to contact all families with a child in the specified age range through a visit by an Indigenous interviewer. Families were presented with a traditional gift and invited to participate. For those families that participated, each family member received $\$ 40$. The recruitment procedure resulted in an overall response rate of $79.4 \%$.

Eight waves of data were collected via yearly interviews with the adolescent and at least one primary caretaker. For these analyses, we used Waves 1, 4, 6, and 8, when diagnostic data were collected, and aligned participants by their age at each wave. The present study includes the 673 adolescents who completed baseline (Wave 1) diagnostic interviews (mean age $[S D]=11.1$ [0.82] years; $50.3 \%$ female). Approximately $11 \%$ lived in a remote location (compared to a rural location), and $85.5 \%$ lived on a reservation/reserve. Average annual per capita family income was $\$ 5,448$, and median parent education level was high school diploma or general equivalence diploma (GED). Retention rates were high across the 8 years of the study, with $87.7 \%$ retention at Wave $4,88 \%$ at Wave 6 , and $78.5 \%$ at Wave 8. Additional details regarding the sample and study methodology are available in Whitbeck et al. ${ }^{12}$

\section{Measures}

$C D$ and AUD Symptoms. Conduct disorder (CD) symptoms and alcohol use disorder (AUD) symptoms, the focus of the study, were assessed via the DSM-IV Diagnostic Interview Schedule for Children-Revised (DISC-R). Trained community interviewers administered the survey. The DISC-R is commonly used for children and adolescents aged 11 years and older. ${ }^{20,21}$ For $\mathrm{CD}$, youth reported whether they had engaged in 14 different behaviors (DSM-IV criteria specify 15 items, but the question regarding forced sex was not included in our study) in the past year. For AUD, youth were asked a series of questions regarding their use of alcohol in the past year, and 11 symptoms were calculated from their responses to assess abuse and dependence. All symptoms were coded as 0 if the symptom was not present and as 1 if the symptom was present. For each disorder, responses were summed into symptom counts to calculate a continuous severity measure. We used youth-reported symptoms at Waves $1,4,6$, and 8 to calculate symptom trajectories based on adolescents' age at each wave, as described below. Descriptive statistics for the disorder symptoms at each age are provided in Table S1, available online.

Wave 1 (Ages 10-12) Risk and Protective Factors. Eight predictor variables (Table S2, available online) were included in the profile analysis, all drawn from Wave 1. Adolescent gender was coded $1=$ female and $0=$ male. Per capita family income was a continuous measure of caretaker-reported household income divided by the number of people living in the household. ${ }^{22}$ Caretaker warmth and support was a mean score of adolescent responses to five items assessing how often someone in their family was warm and supportive $(0=$ none of the time, $1=$ some of the time, $2=$ all of the time). Positive school adjustment was measured with seven yes/no questions regarding positive attitudes toward school; affirmative responses were summed. Discrimination was assessed with 10 items from an adapted version of the Schedule of Racist Events, ${ }^{23}$ measuring how often adolescents experienced negative treatment from others because of their indigenous culture. "Deviant peers" was a summed average of how many of respondents' three best friends engaged in seven behaviors (e.g., drinking, getting into trouble with police). Pastyear caretaker major depressive disorder (MDD) and SUD were assessed using the University of Michigan Composite International Diagnostic Interview ${ }^{24}$ to determine whether any primary caretakers met criteria for past-year MDD or alcohol abuse, drug abuse, or alcohol dependence.

Wave 8 (Ages 17-20) Outcomes. Four dichotomous variables (Table S2, available online) were used in the outcome analysis, all drawn from the final wave of the study. These variables included whether adolescents had graduated or were still attending high school, reported early parenthood (i.e., had a child during the study), had sex under the influence (i.e., engaged in sex in the past year while under the influence of drugs or alcohol), or were arrested in the past year. All variables were coded as present (1) or absent (0).

\section{Data Analyses}

Group-based trajectory modeling using Stata traj was used to identify clusters of adolescents following similar patterns over time for CD and AUD symptoms. ${ }^{25,26}$ Because symptom counts were used, zero-inflated Poisson distributions were specified. We began by modeling CD and AUD symptoms separately to select the appropriate number and shape of trajectory groups. Model selection 
FIGURE 1 Trajectories of conduct and alcohol use disorder symptoms between ages 10 and 19 years. Note: $95 \% \mathrm{Cls}$ are shown in dotted lines.

\section{a Conduct disorder symptoms}

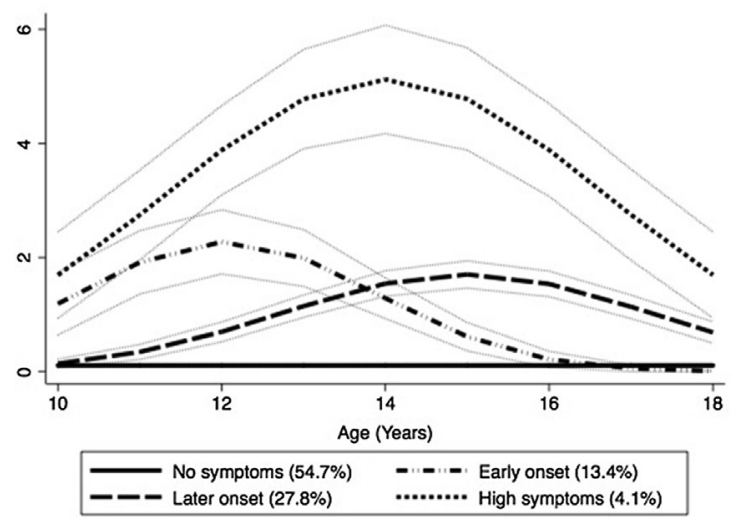

b Alcohol use disorder symptoms

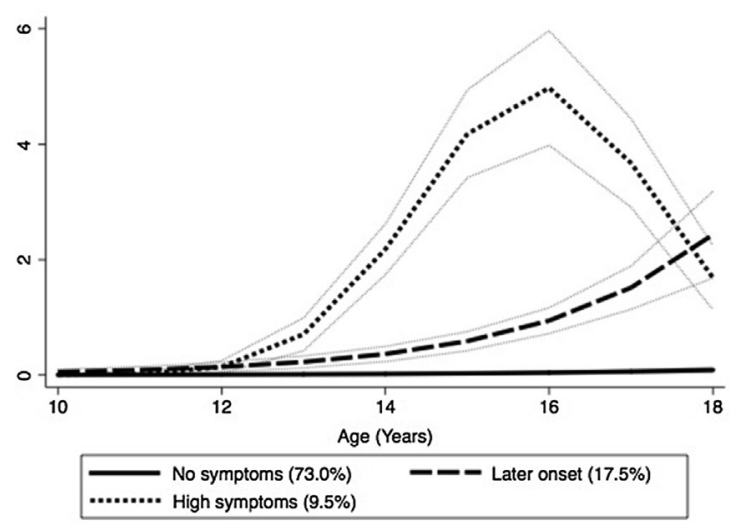

was guided by balancing fit and parsimony-that is, by the lowest Bayesian Information Criterion (BIC) values-as well as substantive interpretation. ${ }^{27,28}$ Each trajectory was fit iteratively including constant, slope, and quadratic terms to determine the best fit to the rate of change represented in the data (i.e., constant symptom levels, linear, or nonlinear change, respectively). Individuals were assigned to trajectory groups for CD and AUD symptoms based on their highest posterior probabilities of group membership, with larger probabilities indicating better fit.

We subsequently created joint posterior probabilities groups that represented the intersection of these groups (i.e., individuals' $\mathrm{CD}$ and AUD group memberships), excluding groups that represented $\leq 1 \%$ of the sample. These joint posterior probabilities were used to create profiles of each group's baseline demographics and risk/protective factors at wave 1 (age 10-12 years), and associated outcomes at wave 8 (age 17-20 years). We used analyses of variance and $\chi^{2}$ tests to compare means and proportions of the risk/ protective factors in each joint trajectory group, and used logistic regressions to compare the trajectory groups on their prediction of the (binary) outcomes at wave 8 , with the asymptomatic trajectory as the reference group.

\section{RESULTS}

\section{CD and AUD Trajectory Groups}

For CD symptom trajectories, the model with four groups fit best; although the BIC value for five groups was smaller, the fifth group resulted in overextraction with less than $2 \%$ of the sample (Table S3, available online). Average posterior probabilities were acceptable (0.77-0.87 for CD). For AUD symptoms, a three-group model was selected because increasing the number of groups to four split the smallest group into two and led to instability in subsequent analyses. Furthermore, average posterior probabilities for the threegroup model were high $(0.82-0.93)$, providing small classification error.

Figure 1 displays the four trajectories of CD symptoms (panel a) and the three trajectories of AUD symptoms (panel b). The best-fitting model of CD symptoms had an intercept term for the first group and quadratic terms for the remaining three groups. AUD symptoms had linear terms for the first two groups and a quadratic term for the third. For both disorders, the largest group was characterized by no symptoms from ages 10 to 18 years ( $54.7 \%$ for CD, and $73 \%$ for AUD). There were also other developmental similarities between

TABLE 1 Joint and Conditional Probabilities of Conduct Disorder (CD) and Alcohol Use Disorder (AUD) Symptoms

\begin{tabular}{|c|c|c|c|c|}
\hline \multirow[b]{2}{*}{$\begin{array}{l}\text { Trajectory of AUD } \\
\text { Symptoms }\end{array}$} & \multicolumn{4}{|c|}{ Trajectory of CD Symptoms } \\
\hline & $\begin{array}{c}\text { No } \\
\text { Symptoms } \\
\text { (n=368) }\end{array}$ & $\begin{array}{c}\text { Early } \\
\text { Onset } \\
(\mathbf{n}=90)\end{array}$ & $\begin{array}{c}\text { Later } \\
\text { Onset } \\
\text { (n= 187) }\end{array}$ & $\begin{array}{l}\text { High } \\
\text { Symptoms } \\
\text { (n=28) }\end{array}$ \\
\hline & \multicolumn{4}{|c|}{ Probability of joint AUD and CD symptoms ${ }^{a}$} \\
\hline $\begin{array}{l}\text { No symptoms } \\
(\mathrm{n}=491)\end{array}$ & 0.53 & 0.09 & 0.11 & 0.00 \\
\hline $\begin{array}{l}\text { Later onset } \\
\qquad(\mathrm{n}=118)\end{array}$ & 0.01 & 0.03 & 0.11 & 0.02 \\
\hline \multirow{2}{*}{$\begin{array}{l}\text { High symptoms } \\
\quad(\mathrm{n}=64)\end{array}$} & 0.01 & 0.01 & 0.06 & 0.02 \\
\hline & \multicolumn{4}{|c|}{$\begin{array}{l}\text { Probability of AUD symptoms conditional on } \\
\text { CD symptoms }{ }^{\text {b }}\end{array}$} \\
\hline $\begin{array}{l}\text { No symptoms } \\
(\mathrm{n}=491)\end{array}$ & 0.97 & 0.68 & 0.39 & 0.00 \\
\hline $\begin{array}{l}\text { Later onset } \\
\qquad(\mathrm{n}=118)\end{array}$ & 0.02 & 0.24 & 0.41 & 0.46 \\
\hline \multirow{2}{*}{$\begin{array}{l}\text { High symptoms } \\
\qquad(\mathrm{n}=64)\end{array}$} & 0.01 & 0.08 & 0.20 & 0.54 \\
\hline & \multicolumn{4}{|c|}{$\begin{array}{c}\text { Probability of CD symptoms conditional on } \\
\text { AUD symptoms }{ }^{c}\end{array}$} \\
\hline $\begin{array}{l}\text { No symptoms } \\
(\mathrm{n}=491)\end{array}$ & 0.73 & 0.12 & 0.15 & 0.00 \\
\hline $\begin{array}{l}\text { Later onset } \\
\qquad(\mathrm{n}=118)\end{array}$ & 0.05 & 0.19 & 0.65 & 0.11 \\
\hline $\begin{array}{l}\text { High symptoms } \\
\quad(\mathrm{n}=64)\end{array}$ & 0.06 & 0.11 & 0.60 & 0.23 \\
\hline \multicolumn{5}{|c|}{$\begin{array}{l}\text { Note: } \\
\text { aThe sum of the cells is } 1 \text { after rounding. } \\
{ }^{b} \text { Each column sums to } 1 \text {. } \\
{ }^{C} \text { Each row sums to } 1 \text {. }\end{array}$} \\
\hline
\end{tabular}


the two disorders. First, each disorder had a group characterized by a later age of onset $(27.8 \%$ for CD, and $17.5 \%$ for AUD), although the onset of symptoms among the lateronset CD group occurred earlier and peaked at approximately age 15 years, whereas the later-onset AUD group continued to increase in the number of symptoms through the end of the study. Second, each disorder also had a smaller group characterized by higher numbers of symptoms in adolescence that declined before age 18 years $(4.1 \%$ for CD, and $9.5 \%$ for AUD). As was the case for the later-onset groups, this highest symptom trajectory emerged and peaked earlier for CD compared to AUD. The fourth CD group, early-onset $(13.4 \%)$, was characterized by an increasing number of symptoms between ages 10 and 12, followed by a decrease.

\section{Joint Trajectory Analyses}

As mentioned above, individuals were stratified into groups based on their joint trajectories of CD and AUD to delineate comorbid and non-comorbid symptom trajectories, summarized as the joint and conditional probabilities of symptom group membership (Table 1 ). The vast majority of the sample (97\%) fell into eight joint trajectory groups that each comprised more than $1 \%$ of the sample. Joint probabilities represent the proportion of adolescents estimated to belong simultaneously to CD and AUD trajectories; conditional probabilities represent the proportion of youth transitioning from CD trajectories to AUD trajectories, and vice versa. Slightly more than half $(53 \%)$ of the sample were in the asymptomatic class for both CD and AUD. Adolescents following the asymptomatic CD trajectory had a 97\% probability of transitioning to the asymptomatic AUD trajectory, and a smaller (73\%) probability of transitioning from the asymptomatic AUD trajectory to the asymptomatic CD trajectory. Only $3 \%$ of adolescents with no presenting CD symptoms ( $2 \%$ of the full sample) went on to develop either later-onset or high AUD symptoms. In contrast, all adolescents with high CD symptoms transitioned into symptomatic AUD trajectories, $46 \%$ of them with later onset AUD and $54 \%$ of them with high AUD symptoms, representing $4 \%$ of the full sample. Most adolescents (68\%) with early-onset CD did not develop subsequent AUD symptoms, in line with the decreasing CD symptoms in this trajectory from age 15 years onward, although $24 \%$ of this group appears to shift in phenotypic expression of externalizing at this age toward later-onset AUD symptoms. Finally, adolescents with lateronset CD symptoms were distributed across AUD trajectories, with $39 \%$ of them transitioning to the no-AUD symptom group, $41 \%$ to the delayed-onset AUD group, and $20 \%$ to the high AUD symptoms group.

\section{Wave 1 (Ages 10-12) Risk and Protective Factors}

There were few statistically significant group differences on sociodemographic factors (Table 2). For example, gender differences between the high CD with later-onset AUD and the asymptomatic group appeared large (75\% male versus $48 \%$ male) but did not reach significance, likely due to the small cell sizes for the high CD groups. In general, exposure to risk factors was lowest among youth in the asymptomatic

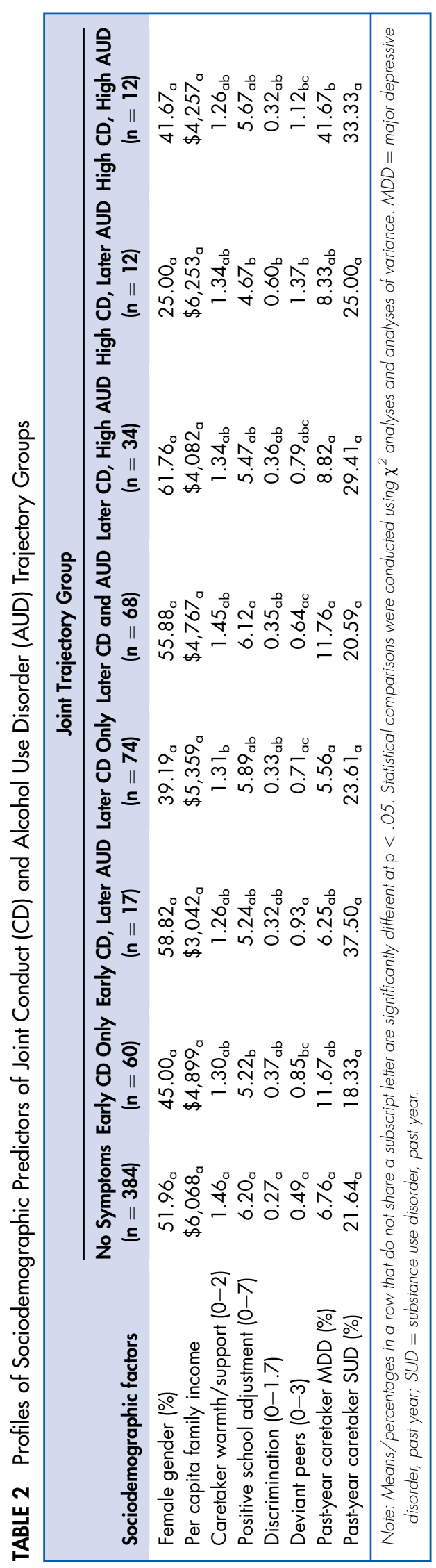

Journal of the American Academy of Child \& Adolescent Psychiatry VOLUME 56 NUMBER 2 FEBRUARY 2017 
TABLE 3 Logistic Regression Analyses of Joint Conduct (CD) and Alcohol Use Disorder (AUD) Trajectory Groups as Predictors for Wave 8 Outcomes

\begin{tabular}{|c|c|c|c|c|c|c|c|c|}
\hline \multirow[b]{2}{*}{ Joint Trajectory Group ${ }^{a}$} & \multicolumn{2}{|c|}{$\begin{array}{l}\text { Graduated/Still } \\
\text { Attending HS }\end{array}$} & \multicolumn{2}{|c|}{ Early Parenthood } & \multicolumn{2}{|c|}{ Sex Under the Influence } & \multicolumn{2}{|c|}{ Arrest } \\
\hline & OR & $95 \% \mathrm{Cl}$ & OR & $95 \% \mathrm{Cl}$ & OR & $95 \% \mathrm{Cl}$ & OR & $95 \% \mathrm{Cl}$ \\
\hline Early CD only $(n=60)$ & 0.67 & $0.33,1.37$ & 1.49 & $0.73,3.06$ & 1.25 & $0.55,2.87$ & $\mathbf{2 . 8 7} 7^{\star \star}$ & $1.38,5.96$ \\
\hline Early $C D$, later AUD $(n=17)$ & 0.46 & $0.13,1.58$ & 1.45 & $0.38,5.54$ & 0.58 & $0.07,4.64$ & 3.76* & $1.08,13.13$ \\
\hline Later CD only ( $n=74)$ & $0.30^{* * *}$ & $0.16,0.57$ & 1.31 & $0.64,2.65$ & $\mathbf{2 . 9 8} \mathbf{8}^{* *}$ & $1.53,5.82$ & $2.25^{\star}$ & $1.08,4.70$ \\
\hline Later $C D$ and $A U D(n=68)$ & 0.79 & $0.41,1.53$ & 1.48 & $0.78,2.81$ & $4.02^{\star * *}$ & $2.20,7.36$ & $\mathbf{4 . 6 2} 2^{\star * *}$ & $2.49,8.59$ \\
\hline Later $C D$, high AUD $(\mathrm{n}=34)$ & $0.23^{\star * *}$ & $0.11,0.52$ & 1.61 & $0.64,4.00$ & $6.95^{\star \star \star}$ & $2.83,17.08$ & 4.70*** & $1.98,11.16$ \\
\hline High $C D$, later AUD $(\mathrm{n}=12)$ & 0.46 & $0.11,1.89$ & 3.49 & $0.91,13.40$ & 4.34* & $0.94,20.09$ & 2.15 & $0.43,10.75$ \\
\hline High CD and AUD $(\mathrm{n}=12)$ & 0.80 & $0.16,3.97$ & 2.18 & $0.53,8,98$ & $7.24^{\star \star}$ & $1.87,28.03$ & $15.03^{\star * *}$ & $3.60,62.80$ \\
\hline
\end{tabular}

group and higher among the youth in the earlier-onset and more symptomatic trajectory groups. For example, the high CD with later-onset AUD group had significantly poorer school adjustment, higher discrimination, and more deviant peers than the asymptomatic group. Youth in the high CD, high SUD group were also significantly more likely than those in the no symptoms groups to have a caretaker with past-year major depression.

\section{Wave 8 (Ages 17-20) Outcomes}

Table 3 presents Wave 8 outcomes for each symptomatic group compared to the asymptomatic group. All symptomatic groups had lower odds (odds ratio $[\mathrm{OR}]=0.23-0.80$ ) of graduating or still attending high school compared to the asymptomatic group, but these differences reached significance only for two later-onset CD groups (with no AUD and high AUD symptoms). Similarly, all symptomatic groups had higher odds (OR $=1.31-3.49)$ of early parenthood-particularly for the high CD groups with comorbid AUD-but these differences did not reach statistical significance. The odds of having sex under the influence of alcohol or drugs were significantly higher $(\mathrm{OR}=2.98-7.24)$ for all of the adolescents with later-onset $\mathrm{CD}$ or high $\mathrm{CD}$ symptoms, among whom the severity of co-occurring AUD was associated with higher odds. Finally, all symptomatic groups had higher odds (OR $=2.15-15.03)$ of being arrested, compared to the asymptomatic group, and these differences reached significance for all groups except for the high $\mathrm{CD} /$ later-onset AUD group.

\section{DISCUSSION}

This study aimed to characterize the developmental trajectories of comorbid and non-comorbid CD and AUD, as well as their associated risk factors and outcomes, over an 8-year time span in a sample of Indigenous youth. Using groupbased trajectory modeling, we identified clusters of adolescents who followed distinct but related trajectories of AUD and CD symptoms between ages 10 and 18 years. Based on the development of $\mathrm{CD}$ and AUD symptoms, adolescents fell into eight common groups that represented $97 \%$ of the sample. Although demographic and risk factors did not statistically significantly differentiate these groups (likely due to the small sizes of some of the groups that limited our ability to achieve standard significance levels), some systematic patterns were evident. The groups characterized by earlier onset and/or higher levels of symptoms tended to have a greater concentration of risk factors. These groups did effectively predict negative outcomes in late adolescence. These results are discussed in the context of the literature below.

We found four trajectory groups for $C D$, including early onset, later onset, and high mid-adolescence symptoms, as well as an asymptomatic group that characterized the majority $(55 \%)$ of the sample throughout adolescence. Four similar trajectory groups were also found in the Dunedin Multidisciplinary Health and Development Study, which included primarily white participants in New Zealand. ${ }^{29}$ In our study, more youth were in the low CD symptoms group than in the New Zealand study (55\% versus $46 \%$ ), whereas fewer youth were in the high/persistent CD symptoms group than in that study (4\% versus $11 \%$ ). This low $/$ no symptom group was a larger group than found for this sample previously, likely due to the use of a smaller range of CD aggressive symptoms. ${ }^{30}$

The proportion of our sample that reported no CD symptoms also increased over time, with $68 \%$ of the sample in a group characterized by no CD symptoms at age 18 years; similarly, $17.5 \%$ of the sample reported CD symptoms at age 10 years, increasing to $32 \%$ of the sample at age 18 years. In sum, a majority and comparatively higher proportion of Indigenous youth in this study were without symptoms of conduct disorder relative to previously published reports with non-Native youth. ${ }^{29}$ This finding is particularly striking, given the disproportionate exposure to contemporary and historical risk factors for many Indigenous youth.

We also found three clusters for AUD, including lateonset and high mid-adolescence trajectories that mirrored the developmental trajectories of the CD groups. Nearly 
three-fourths (73\%) of the sample reported no AUD symptoms, in line with high rates of non-use among American Indians in general. ${ }^{19}$ Notably, the earliest-emerging symptoms were from age 12 years onward for AUD, whereas $17.5 \%$ of the sample reported CD symptoms at age 10 years, as mentioned above. This suggests that CD symptoms preceded the development of AUD symptoms. Prior work with these data examined longitudinal trajectories of alcohol and substance use (i.e., alcohol, marijuana, or cigarettes) and found that substance use behaviors emerged earlier in development than diagnostic symptoms. ${ }^{31}$ As such, it is possible that some of the adolescents were already using alcohol at age 10 years, but did not yet meet diagnostic criteria for AUD.

When we examined the joint probabilities of membership in the developmental trajectories across the two disorders, systematic patterns of comorbidity became clear. For example, the overwhelming majority (97\%) of adolescents reporting no $\mathrm{CD}$ symptoms also did not report any AUD symptoms, such that more than half of the sample was asymptomatic for both disorders throughout adolescence. This suggests that the absence of early-presenting CD symptoms highlights a corresponding low risk for subsequent AUD symptoms to develop. The next highest risk was the early-onset CD group, in which $68 \%$ reported no $C D$ or AUD symptoms by age 16 years, but $24 \%$ shifted into the late-onset AUD symptom trajectory. Following this, the later-onset CD group represented the next highest risk, with $59 \%$ reporting concurrent or subsequent symptoms. As expected, the CD group with the greatest number of symptoms across the study period represented the highest risk, with all of the adolescents who reported high CD behaviors also reporting subsequent AUD symptoms.

Our joint trajectory analyses of AUD and CD revealed eight groups that comprised $97 \%$ of the sample, which we compared with respect to reported risk factors at the start of the study, and negative associated outcomes at the end of the study. When compared to the other symptomatic groups, at age 10 to 12 years (baseline), this asymptomatic group had greater caretaker warmth and support, positive school adjustment, less discrimination, and fewer deviant peers, and were less likely to have a caretaker with major depression. These findings correspond with the literature on caretaker warmth $^{32}$ and depression ${ }^{33}$ as two potent factors (in opposite directions) when considering adolescent psychopathology.

A variety of negative late-adolescent outcomes were systematically related to prior CD, AUD, and the comorbidity between them. Aging out of early CD symptoms (i.e., the early-onset $\mathrm{CD}$ group) tended to be associated with lower odds of negative outcomes in late adolescence, in line with the corresponding early decline and apparently adolescence-limited CD trajectory in this group ${ }^{5}$; later-onset $\mathrm{CD}$ represented the next highest risk, followed by the group with high levels of CD behaviors, as expected. The severity of co-occurring AUD symptoms also compounded this risk, predicting still higher odds for nearly every negative outcome. These outcomes have significant implications for opportunities in later life. Although not statistically significant, we found that youth experiencing comorbid AUD/CD and earlier onset of either/both had higher odds of becoming parents in adolescence. Similar to prior research, AUD/CD were associated with greater odds of having sex under the influence. ${ }^{34}$ Above, we noted that youth with caregivers experiencing SUD or MDD were more likely to experience comorbid symptoms themselves. Taken together, these findings illustrate the potential for intergenerational cycles of behavioral and mental health problems.

This study had a large sample size with good follow-up rates that shed light on the prospective relationship between CD and AUD symptoms, including predictors and outcomes of different comorbid trajectories. Prior studies have not considered CD and AUD concurrently. In line with calls for dimensional models of diagnostic criteria, we used a summed total of DSM-IV symptoms instead of a present/ absent approach. ${ }^{35}$ Finally, this study extends the developmental literature to include indigenous groups. At the same time, results are limited in that they were drawn from a single indigenous cultural group.

In summary, the development and level of CD symptoms consistently predicted the development and level of subsequent AUD symptoms, suggesting that $\mathrm{CD}$ behaviors reflect an early-emerging indicator of disinhibition (i.e., the core of externalizing psychopathology $)^{16}$ in this sample of American Indian and First Nations youth. The severity of the joint symptom trajectories was systematically associated with negative outcomes in late adolescence, and some patterns of early risk and protective factors emerged. Given substantial risk factors, the youth displayed significant resilience in terms of prevalence of comorbid CD-SUD. Future studies should examine the continuity or change in symptomatology into adulthood and identify the longer-term consequences of early and increasing symptoms such as impaired or delayed transition into adult roles. $\varepsilon$

Accepted November 22, 2016

Drs. Greenfield and Walls are with University of Minnesota Medical School, Duluth campus. Dr. Sittner is with Oklahoma State University, Stillwater. Dr. Forbes is with University of Minnesota, Twin Cities. Dr. Whitbeck is with University of Nebraska-Lincoln.

The authors acknowledge funding from the following sources: National Institute on Drug Abuse 13580 (L.W.I, 039912 (M.W.), and T32 037183 (B.G., M.F.), National Institute of Mental Health 67281 (L.W.), and National Institute on Alcohol Abuse and Alcoholism 020299 (L.W.).

This study was presented as an abstract at the 4th annual meeting of the American Psychological Association Division 50: Collaborative Perspectives on Addiction, San Diego, CA, March 18-19, 2016, and the Native Children's Research Exchange II 2016, Denver, CO, September 29-30, 2016.

Drs. Sittner and Forbes served as the statistical experts for this research.

The authors thank the communities and participants who shared their time and experiences for this project and the reviewers who offered helpful feedback.

Disclosure: Drs. Greenfield, Sittner, Forbes, Walls, and Whitbeck report no biomedical financial interests or potential conflicts of interest.

Correspondence to Brenna L. Greenfield, PhD, University of Minnesota Medical School, Duluth Campus, 335 SMed, 1035 University Drive, Duluth, MN 55812; e-mail: green970@d.umn.edu

0890-8567/\$36.00/@2016 American Academy of Child and Adolescent Psychiatry

hitp://dx.doi.org/10.1016/i.jaac.2016.11.009 
REFERENCES

1. Harford TC, Yi H-Y, Chen CM, Grant BF. Psychiatric Symptom Clusters as Risk Factors for Alcohol Use Disorders in Adolescence: a national study. Alcohol Clin Exp Res. 2015;39:1174-1185.

2. Hopfer C, Salomonsen-Sautel S, Mikulich-Gilbertson S, et al. Conduct disorder and initiation of substance use: a prospective longitudinal study. J Am Acad Child Adolesc Psychiatry. 2013;52:511-518.

3. Cerdá M, Bordelois PM, Keyes KM, Galea S, Koenen KC, Pardini D. Cumulative and recent psychiatric symptoms as predictors of substance use onset: does timing matter? Addiction. 2013;108:2119-2128.

4. Kessler RC, Avenevoli S, McLaughlin KA, et al. Lifetime co-morbidity of DSM-IV disorders in the US National Comorbidity Survey Replication Adolescent Supplement (NCS-A). Psychol Med. 2012;42:1997-2010.

5. Moffitt TE. Adolescence-limited and life-course-persistent antisocial behavior: a developmental taxonomy. Psychol Rev. 1993;100:674-701.

6. Loeber R, Burke JD, Lahey BB, Winters A, Zera M. Oppositional defiant and conduct disorder: a review of the past 10 years, part I. J Am Acad Child Adolesc Psychiatry. 2000;39:1468-1484.

7. Boden JM, Fergusson DM, Horwood LJ. Risk factors for conduct disorder and oppositional/defiant disorder: evidence from a New Zealand birth cohort. J Am Acad Child Adolesc Psychiatry. 2010;49:1125-1133.

8. Costello EJ, Farmer EM, Angold A, Burns BJ, Erkanli A. Psychiatric disorders among American Indian and white youth in Appalachia: the Great Smoky Mountains Study. Am J Public Health. 1997;87:827-832.

9. Whitbeck LB, Sittner Hartshorn KJ, Walls ML. Indigenous Adolescent Development: Psychological, Social and Historical Contexts. New York: Routledge/Taylor and Francis Group; 2014.

10. Alegria M, Vallas M, Pumariega AJ. Racial and ethnic disparities in pediatric mental health. Child Adolesc Psychiatr Clin N Am. 2010;19: 759-774.

11. Beals J, Piasecki J, Nelson S, et al. Psychiatric disorder among American Indian adolescents: prevalence in Northern Plains youth. J Am Acad Child Adolesc Psychiatry. 1997;36:1252-1259.

12. Whitbeck LB, Sittner Hartshorn KJ, Crawford DM, Walls ML, Gentzler KC, Hoyt DR. Mental and substance use disorders from early adolescence to young adulthood among indigenous young people: final diagnostic results from an 8-year panel study. Soc Psychiatry Psychiatr Epidemiol. 2014;49:961-973.

13. Armstrong TD, Costello EJ. Community studies on adolescent substance use, abuse, or dependence and psychiatric comorbidity. J Consult Clin Psychol. 2002;70:1224-1239.

14. Wright AGC, Simms LJ. A metastructural model of mental disorders and pathological personality traits. Psychol Med. 2015;45:2309-2319.

15. Grant JD, Lynskey MT, Madden PA, et al. The role of conduct disorder in the relationship between alcohol, nicotine and cannabis use disorders. Psychol Med. 2015;45:3505-3515.

16. Krueger RF, South SC, Achenbach TM, et al. Externalizing disorders: Cluster 5 of the proposed meta-structure for DSM-V and ICD-11. Psychol Med. 2009;39:2061.

17. Krueger RF, Markon KE, Patrick CJ, Benning SD, Kramer MD. Linking antisocial behavior, substance use, and personality: an integrative quantitative model of the adult externalizing spectrum. J Abnorm Psychol. 2007;116:645-666.
18. Chartier K, Caetano R. Ethnicity and health disparities in alcohol research. Alcohol Res Health. 2010;33:152-160. Available at: http:/ /www.ncbi.nlm. nih.gov/pubmed/21209793. Accessed September 24, 2016.

19. Cunningham JK, Solomon TA, Muramoto ML. Alcohol use among Native Americans compared to whites: examining the veracity of the "Native American elevated alcohol consumption" belief. Drug Alcohol Depend. 2016;160:65-75.

20. Shaffer D, Schwab-Stone M, Fisher $\mathrm{P}$, et al. The Diagnostic Interview Schedule for Children-Revised Version (DISC-R): I. Preparation, field testing, interrater reliability, and acceptability. J Am Acad Child Adolesc Psychiatry. 1993;32:643-650.

21. Shaffer D, Schwab-Stone M, Fisher P. A revised version of the Diagnostic Interview Schedule for Children (DISC-R): results of a field trial and proposals for a new instrument (DISC-2). Report submitted to the National Institute of Mental Health. 1988

22. Conger R, Elder GH. Families in Troubled Times: Adapting to Change in Rural America. Piscataway, NJ: Aldine Transaction; 1994.

23. Landrine H, Klonoff EA. The Schedule of Racist Events: A Measure of Racial Discrimination and a Study of Its Negative Physical and Mental Health Consequences. J Black Psychol. 1996;22:144-168.

24. World Health Organization. Composite International Diagnostic Interview (CIDI), Version 1.0. Geneva, Switzerland: WHO; 1990.

25. Nagin DS, Tremblay RE. Analyzing developmental trajectories: a semiparametric, group-based approach. Psychol Methods. 1999;4:139-157.

26. Jones BL, Nagin DS. A note on a Stata plugin for estimating group-based trajectory models. Sociol Methods Res. 2013;42:608-613.

27. Nagin DS. Group-Based Modeling of Development. Cambridge, MA: Harvard University Press; 2005.

28. Nagin DS, Odgers CL. Group-based trajectory modeling in clinical research. Annu Rev Clin Psychol. 2010;6:109-138.

29. Odgers CL, Moffitt TE, Broadbent JM, et al. Female and male antisocial trajectories: from childhood origins to adult outcomes. Dev Psychopathol. 2008;20:673-716

30. Sittner KJ, Hautala D. Aggressive delinquency among north American indigenous adolescents: trajectories and predictors. Aggress Behav. 2016; 42:274-286.

31. Sittner KJ. Trajectories of substance use: onset and adverse outcomes among north American Indigenous adolescents. J Res Adolesc. 2015;26: 830-844.

32. Hay C, Meldrum RC, Widdowson AO, Piquero AR. Early aggression and later delinquency: considering the redirecting role of good parenting. Youth Violence Juv Justice. [Epub ahead of print] March 1, 2016: 1541204016631805 .

33. Goodman SH, Rouse MH, Connell AM, Broth MR, Hall CM, Heyward D. Maternal depression and child psychopathology: a meta-analytic review. Clin Child Fam Psychol Rev. 2011;14:1-27.

34. Wu J, Witkiewitz K, McMahon RJ, Dodge KA. A parallel process growth mixture model of conduct problems and substance use with risky sexual behavior. Drug Alcohol Depend. 2010;111:207-214.

35. Hudziak J, Achenbach T, Althoff R, Pine DS. A dimensional approach to developmental psychopathology. Int J Methods Psychiatr Res. 2007;16: S16-S23. 
TABLE S1 Descriptive Statistics of Alcohol Use Disorder and Conduct Disorder Symptoms ( $\mathrm{n}=673$ )

\begin{tabular}{|c|c|c|c|c|c|c|c|c|c|c|}
\hline & \multicolumn{5}{|c|}{ Alcohol Use Disorder Symptoms } & \multicolumn{5}{|c|}{ Conduct Disorder Symptoms } \\
\hline & $\mathbf{n}$ & Mean & SD & Min & Max & $\mathbf{n}$ & Mean & SD & Min & Max \\
\hline Age $10 y$ & 171 & 0.01 & 0.08 & 0.00 & 1.00 & 171 & 0.35 & 0.97 & 0.00 & 8.00 \\
\hline Age $11 y$ & 272 & 0.01 & 0.06 & 0.00 & 1.00 & 272 & 0.48 & 1.21 & 0.00 & 12.00 \\
\hline Age $12 y$ & 212 & 0.01 & 0.12 & 0.00 & 4.00 & 212 & 0.71 & 1.26 & 0.00 & 7.00 \\
\hline Age $13 y$ & 173 & 0.11 & 0.48 & 0.00 & 8.00 & 173 & 0.83 & 1.68 & 0.00 & 11.00 \\
\hline Age 14 y & 239 & 0.48 & 1.35 & 0.00 & 8.00 & 239 & 1.00 & 1.76 & 0.00 & 11.00 \\
\hline Age $15 y$ & 332 & 0.40 & 1.24 & 0.00 & 11.00 & 332 & 0.72 & 1.29 & 0.00 & 11.00 \\
\hline Age $16 y$ & 257 & 0.79 & 1.91 & 0.00 & 10.00 & 257 & 0.71 & 1.39 & 0.00 & 9.00 \\
\hline Age 17 y & 316 & 0.52 & 1.44 & 0.00 & 11.00 & 316 & 0.50 & 1.09 & 0.00 & 6.00 \\
\hline Age $18 y$ & 230 & 0.70 & 1.62 & 0.00 & 7.00 & 230 & 0.29 & 0.81 & 0.00 & 6.00 \\
\hline
\end{tabular}

TABLE S2 Descriptive Statistics for Profile and Outcome Variables

\begin{tabular}{|llllll|}
\hline Variable & $\mathbf{n}$ & Mean/\% & SD & Min & Max \\
Profile variables (Wave 1) & & & & \\
Gender (female = 1) & 672 & $50.30 \%$ & 0.50 & 0 & 1 \\
Per capita family income & 653 & 5.54 & 4.04 & 0.25 & 25 \\
Caretaker warmth and support 671 & 1.41 & 0.37 & 0 & 2 \\
Positive school adjustment & 671 & 5.97 & 1.46 & 0 & 7 \\
Perceived discrimination & 666 & 0.31 & 0.29 & 0 & 1.7 \\
Deviant peers & 669 & 0.62 & 0.58 & 0 & 3 \\
Past-year caretaker MDD & 665 & $10.08 \%$ & 0 & 1 \\
Past-year caretaker SUD & 665 & $22.41 \%$ & 0 & 1 \\
Outcome variables (Wave 8) & & & & \\
Graduating or attending HS & 526 & $75.48 \%$ & 0 & 1 \\
Early parenthood & 528 & $21.97 \%$ & 0 & 1 \\
Sex under the influence & 507 & $23.87 \%$ & 0 & 1 \\
Arrest & 526 & $20.53 \%$ & 0 & 1 \\
\hline Note: Per capita family income in thousands of dollars. HS = high school; \\
Max = maximum; MDD = major depressive disorder; Min = minimum; \\
SUD = substance use disorder. \\
\end{tabular}

TABLE S3 Bayesian Information Criterion (BIC) Values for Model Selection

\begin{tabular}{|lc|}
\hline No. of CD Symptom Groups & BIC \\
1 & -2727.95 \\
2 & -2375.82 \\
3 & -2326.18 \\
4 & -2283.20 \\
5 & -2273.25 \\
No. of AUD Symptom Groups & BIC \\
1 & -1951.88 \\
2 & -1418.96 \\
3 & -1360.89 \\
4 & -1311.14 \\
5 & -1324.17 \\
\hline Note: AUD = alcohol use disorder; CD = conduct disorder; No. $=$ number. \\
\hline
\end{tabular}

